\title{
Finite element modeling of fatigue crack bifurcation
}

\author{
A.C.O. Miranda ${ }^{\text {a }}$, M.A. Meggiolaro ${ }^{\text {b }}$, J.T.P. Castro ${ }^{\text {b,* }}$, L.F. Martha ${ }^{\text {a }}$ \\ ${ }^{a}$ Civil Engineering Department, Pontifical Catholic University, Rio de Janeiro, RJ 22453-900, Brazil \\ ${ }^{b}$ Mechanical Engineering Department, Pontifical Catholic University, Rio de Janeiro, RJ 22453-900, Brazil
}

\begin{abstract}
The influence of overload-induced crack deflections and bifurcations on the propagation behavior of mode I fatigue cracks is studied using specialized finite element (FE) software. The FE program is validated through comparisons between FE-calculated and analytical stress intensity factors (SIF) for a crack with a small kink at its tip. The SIF of bifurcated cracks are then obtained using the software. It is observed that such deviations of the crack path can cause significant growth retardation and even crack arrest.
\end{abstract}

Keywords: Fatigue; Crack propagation; Growth retardation; Bifurcation; Overload; Finite element

\section{Introduction}

Fatigue crack branching is a well-known phenomenon especially in brittle or semi-brittle materials. Much effort has been given to the case of symmetrically bifurcated (forked) cracks [1]. Although many branches can be developed along the main crack path, it is experimentally observed that only the fastest branch continues to grow, while all others are brought to a stop due to the shielding caused by this fastest branch. However, symmetrical models available in the literature cannot account for such effects. In addition, very few results are available for the real case of bifurcated cracks with different branch lengths. In this work, a specialized FE program called Quebra2D is used to calculate the SIF of bifurcated and kinked cracks, allowing for a better understanding of the influence of crack deflection in the propagation life of structural components.

\section{Finite element software description}

The Quebra2D program simulates two-dimensional fracture processes based on a finite-element (FE) self-adaptive mesh-generation strategy, using appropriate crack tip elements and crack increment criteria [2]. The adaptive FE analyses are coupled with modern and efficient automatic remeshing schemes. The meshing algorithm especially developed for Quebra2D works both for regions without

*Corresponding author. Tel.: +55 (21) 2511-5846; Fax: +55 (21) 3114-1165; E-mail: jtcastro@mec.puc-rio.br cracks and for regions with one or multiple cracks, which may be either embedded or surface breaking. The $2 \mathrm{D}$ algorithm has been designed to meet four specific requirements, as follows. First, the algorithm should produce well-shaped elements, avoiding elements with poor aspect ratio. Second, the generated mesh should conform to an existing discretization on the region boundary. Third, the algorithm should shift smoothly between regions with elements of highly varying size, because in crack analysis it is not uncommon for the elements near the crack tip to be two orders of magnitude smaller than the other elements. And fourth, the algorithm should have specific capabilities for modeling cracks, which are usually idealized without volume, i.e. the surfaces representing the two sides of a crack face are distinct, but geometrically coincident. This means that nodes on opposite sides of crack faces may have identical coordinates, and the algorithm must be able to discriminate between the nodes and to select the one on the proper crack side.

In the Quebra2D program, three methods can be chosen to compute the stress intensity factors along the (generally curved) crack path: the displacement correlation technique, the potential energy release rate computed by means of a modified crack-closure integral technique, and the $J$ integral computed by means of the equivalent domain integral (EDI) together with a mode decomposition scheme [2]. The EDI method replaces the $J$-integral along a contour by another one over a finite size domain, using the divergence theorem, which is more convenient for FE analysis. Since Bittencourt et al. [3] showed that for sufficiently refined FE meshes all three methods predict essentially the 
same results, only the EDI method is considered in the calculations.

\section{Finite element software validation}

To validate the Quebra2D program, the modes I and II SIF $k_{1}$ and $k_{2}$ of an infinitesimally kinked crack $(b / a \rightarrow 0$ in Fig. 1a) are obtained and compared to the analytical solutions [4]:

$k_{1}=\frac{1}{4}\left(3 \cos \frac{\alpha}{2}+\cos \frac{3 \alpha}{2}\right) \cdot K_{I}$

$k_{2}=\frac{1}{4}\left(\sin \frac{\alpha}{2}+\sin \frac{3 \alpha}{2}\right) \cdot K_{I}$

where $K_{I}$ is the mode I SIF of the straight crack without the kink. For calculation purposes, a standard CT specimen is FE modeled with width $w=32.0 \mathrm{~mm}$, crack length $a=14.9 \mathrm{~mm}$, and a very small kink with length $b=10 \mu \mathrm{m}$. Eqs. (1) and (2) are independent of $b / a$ for very small ratios such as $10 \mu \mathrm{m} / 14.9 \mathrm{~mm}=0.00067$, therefore the chosen lengths should be appropriate for this validation. Fig. 2 shows a comparison between the analytical and the
FE-predicted $k_{1}$ and $k_{2}$ (normalized by $K_{I}$ ) for several kink angles $\alpha$, showing a very good agreement.

Note that an efficient meshing algorithm is fundamental to avoid elements with poor aspect ratio, since the ratio between the size scale of the larger and smaller elements is above 1,000 in this case. To accomplish that, Quebra2D uses an innovative algorithm incorporating a quadtree procedure to develop local guidelines to generate elements with the best possible shape. The internal nodes are generated simultaneously with the elements, using the quadtree procedure only as a node-spacing function. This approach tends to give a better control over the generated mesh quality and to decrease the amount of heuristic cleaning-up procedures. Moreover, it specifically handles discontinuities in the domain or boundary of the model. Finally, to enhance the quality of the shape of the mesh element, an $a$ posteriori local mesh improvement procedure is used.

\section{Crack bifurcation predictions}

Having validated the FE software, the same CT specimen is then used to model bifurcated cracks with lengths $c=10 \mu \mathrm{m}$ (for the shorter branch) and $b=10$ or $20 \mu \mathrm{m}$

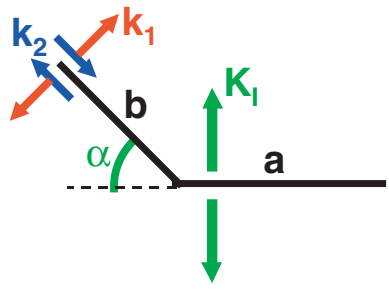

(a)



(b)

Fig. 1. Schematic representation of (a) kinked and (b) bifurcated crack geometries.

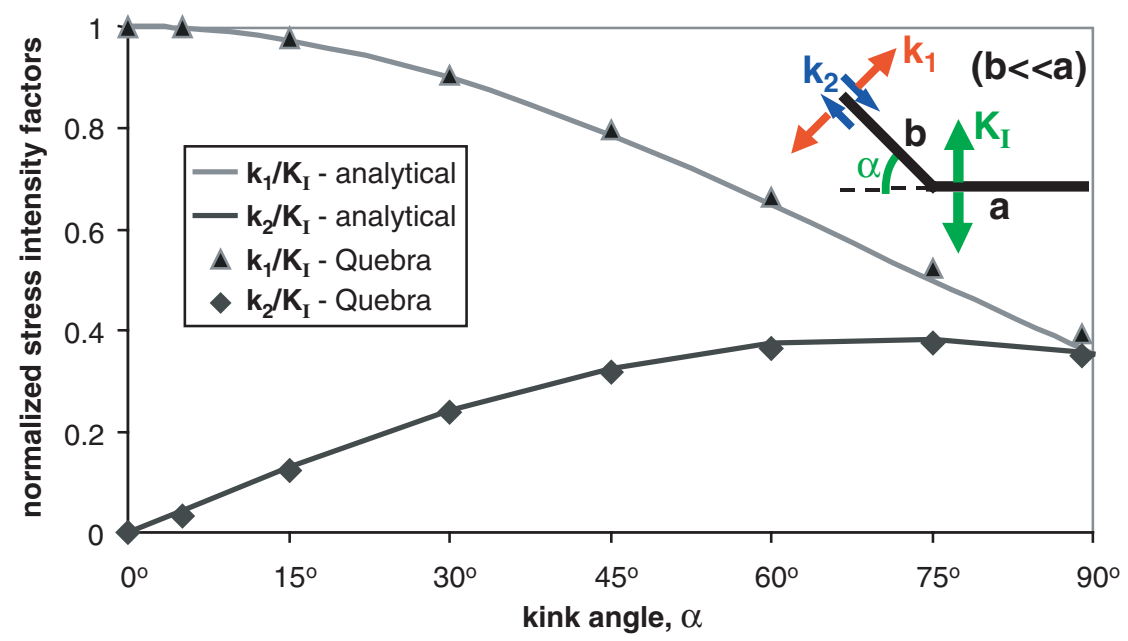

Fig. 2. Validation of the Quebra2D software for a kinked crack. 
(for the longer one), with bifurcation angles $2 \alpha$ between $30^{\circ}$ and $180^{\circ}$ (see Fig. 1b). Note that typical overloadinduced bifurcated cracks can have initial branch lengths between 10 and $100 \mu \mathrm{m}$, with $2 \alpha$ varying between $30^{\circ}$ (for very brittle materials such as glass) and $180^{\circ}$ (in the vicinity of the interface of a bi-material composite, when a crack propagates from the weak to the strong material [5]).

Fig. 3 shows the FE results for the SIF $k_{1}$ and $k_{2}$ (normalized by $K_{I}$ ) of symmetrically and asymmetrically bifurcated cracks. There is a marked increase in the $k_{1}$ and $k_{2}$ SIF for the larger branch (and decrease for the shorter one), if compared to the symmetrically branched solutions. As the length difference between both branches increases, it is expected that the propagation rate of the shorter one is reduced until it arrests, after which the larger branch will dominate. This shielding effect of the longer branch over the shorter one is larger for small bifurcation angles, typically below $120^{\circ}$, see Fig. 3. For large values of $2 \alpha$, the shielding effect is much smaller, as it would be expected since the branch tips are further apart in this case.
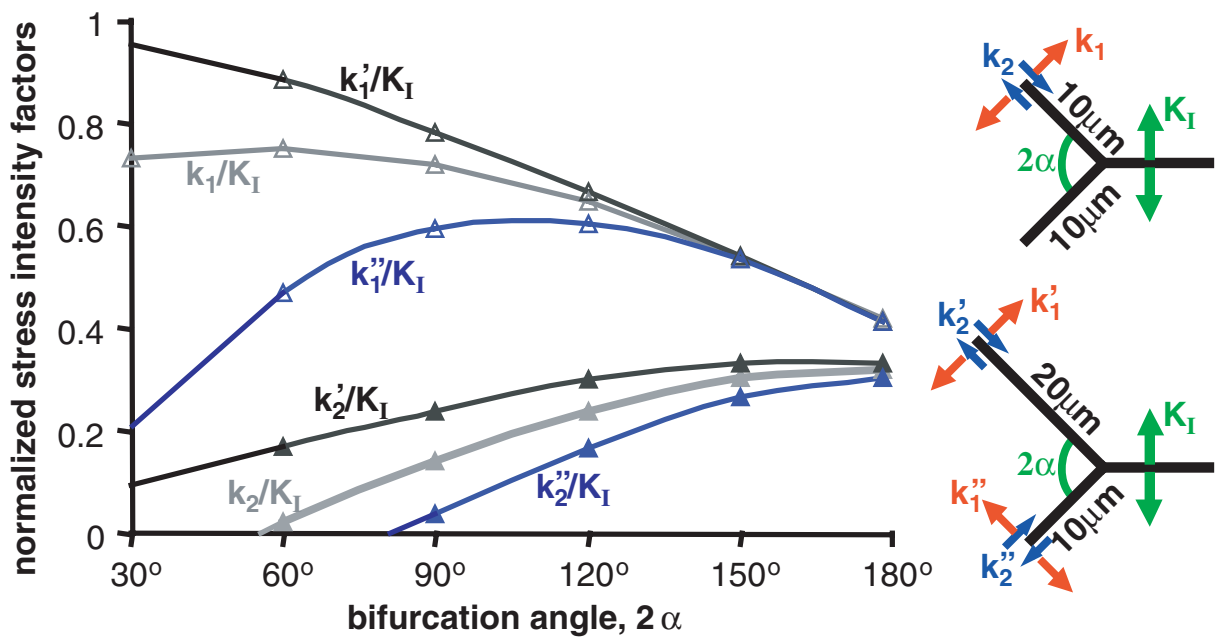

Fig. 3. Stress intensity factors for symmetrically and asymmetrically bifurcated cracks.
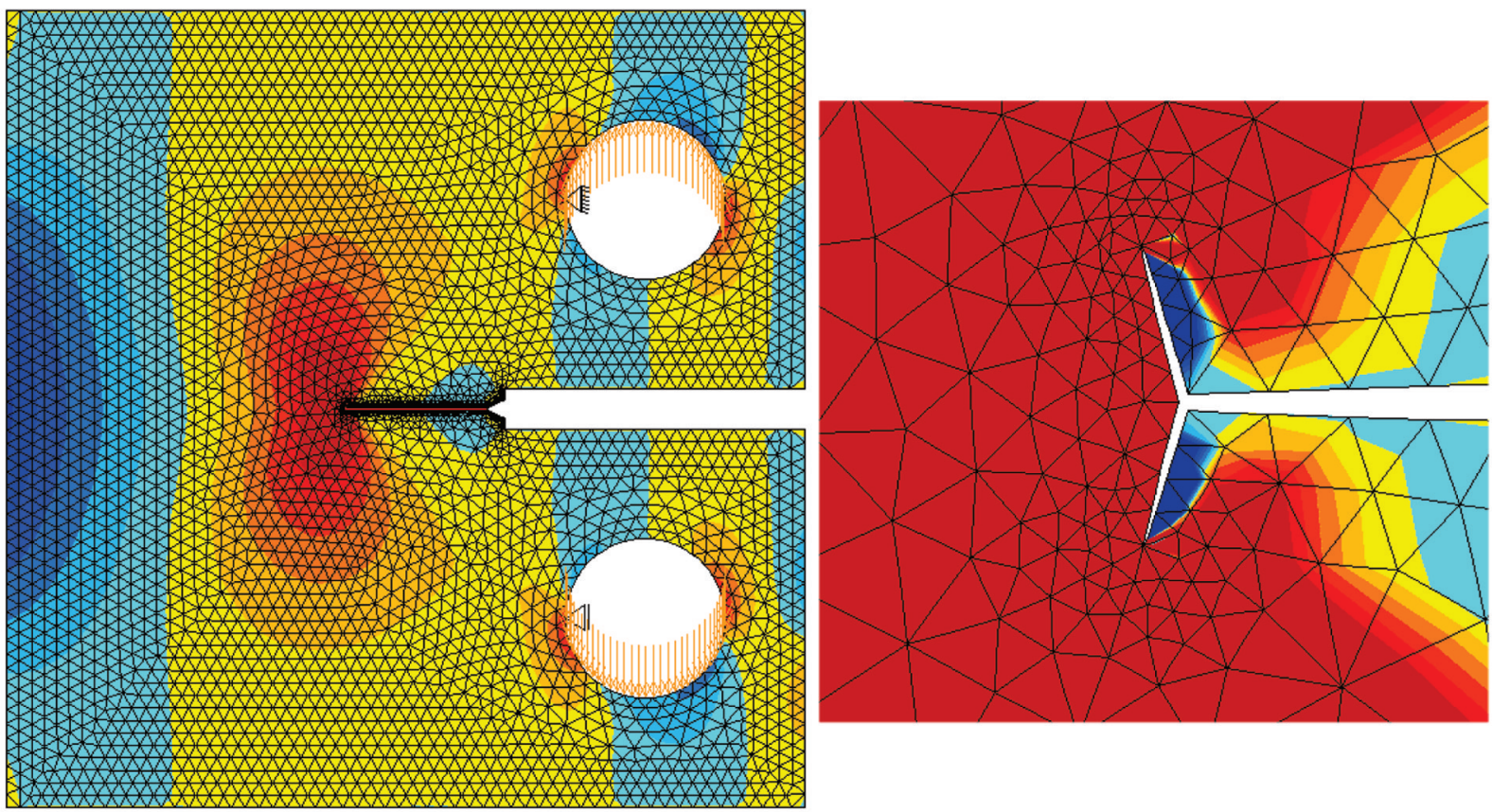

Fig. 4. Finite element results for a CT specimen with a bifurcated crack with angle $2 \alpha=150^{\circ}$ (left) and close-up of the $10 \mu \mathrm{m}$ branches (right). 
Therefore, for larger bifurcation angles the shorter branch is expected to take a longer time to arrest, prolonging the retardation effect.

Fig. 4 shows the contour plots of the stress in the load direction axis, obtained from the FE analysis on a symmetrically bifurcated crack with $2 \alpha=150^{\circ}$.

Finally, it must be pointed out that the obtained FE results might have some limitations, because such small bifurcations can be of a size comparable to the scale of local plasticity (e.g. the plastic zone size) or the microstructural features (e.g. grain size). Also, closure and environmental effects must be subtracted before comparing the bifurcation model predictions with measured crack growth rates [6].

\section{Conclusions}

In this work, planar FE calculations have been performed to estimate the changes in the propagation rates due to overload-induced crack bifurcation. It is found that crack deflection processes alone can significantly reduce the stress intensity factors (SIF) and therefore the propagation rates, leading to crack retardation or even arrest. In particular, the ratio between the bifurcated and the preoverload mode I SIF can be as low as 0.4. Also, very small differences between the lengths of the bifurcated branches are sufficient to induce a much larger SIF and thus crack propagation of the longer branch.

\section{References}

[1] Suresh S. Micromechanisms of fatigue crack growth retardation following overloads. Eng Fract Mech 1983;18(3):577593.

[2] Miranda ACO, Meggiolaro MA, Castro JTP, Martha LF, Bittencourt TN. Fatigue crack propagation under complex loading in arbitrary 2D geometries. In: Braun AA, McKeighan PC, Lohr RD (Eds), Applications of Automation Technology in Fatigue and Fracture Testing and Analysis. ASTM STP 2002;1411(4):120-146.

[3] Bittencourt TN, Wawrzynek PA, Ingraffea AR, Sousa JLA. Quasi-automatic simulation of crack propagation for 2D LEFM problems. Eng Fract Mech 1996;55:321-334.

[4] Suresh S. Fatigue of Materials. Cambridge University Press, 1998.

[5] Pippan R, Flechsig K, Riemelmoser FO. Fatigue crack propagation behavior in the vicinity of an interface between materials with different yield stresses. Mater Sci Eng 2000;A283:225-233.

[6] Suresh S. Crack deflection: implications for the growth of long and short fatigue cracks. Metall Trans 1983;14A:23752385 . 